\title{
GLAD!
}

Revue sur le langage, le genre, les sexualités

05 | 2018

Raconter les sexualités depuis la marge

\section{Marien Gouyon. 2018. Ana Loubia. Ethnographie des homosexualités masculines à Casablanca}

Lucille Gallardo

\section{OpenEdition}

Édition électronique

URL : http://journals.openedition.org/glad/1158

ISSN : 2551-0819

Éditeur

Association GSL

Référence électronique

Lucille Gallardo, «Marien Gouyon. 2018. Ana Loubia. Ethnographie des homosexualités masculines à Casablanca », GLAD! [En ligne], 05 | 2018, mis en ligne le 15 décembre 2018, consulté le 17 décembre 2020. URL : http://journals.openedition.org/glad/1158

Ce document a été généré automatiquement le 17 décembre 2020.

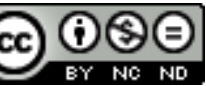

La revue GLAD! est mise à disposition selon les termes de la Licence Creative Commons Attribution Pas d'Utilisation Commerciale - Pas de Modification 4.0 International. 


\title{
Marien Gouyon. 2018. Ana Loubia. Ethnographie des homosexualités masculines à Casablanca
}

\author{
Lucille Gallardo
}

\section{RÉFÉRENCE}

Marien Gouyon. 2018. Ana Loubia. Ethnographie des homosexualités masculines à Casablanca. Vulaines-sur-Seine : Éditions du croquant, "Sociétés et politique en Méditerranée ». 225 pages.

1 Avec Ana Loubia. Ethnographie des homosexualités masculines à Casablanca, Marien Gouyon publie son premier livre issu de sa thèse d'anthropologie sociale soutenue en juillet 2015 à l'École des hautes études en sciences sociales à Paris. Il y propose une analyse de la complexité des constructions homosexuelles identitaires à Casablanca dans une approche à la fois non-culturaliste et «non hégémonique " particulièrement pertinente. Non culturaliste d'une part parce que l'auteur se démarque des travaux qui analysent les masculinités ou les identités et normes sexuelles à partir de traits ou d'épistémologies «culturels » - « arabes » ou " Occidentaux » - qui participent, selon lui, à dénier le caractère identitaire de l'homosexualité au Maroc en confinant les relations homosexuelles à de strictes pratiques. D'autre part, l'auteur se positionne à distance des travaux qui considèrent l'identité homosexuelle uniquement sous son modèle revendicatif et public (ayant pour pilier le coming-out et la lutte pour l'égalité des droits) hérité de l'histoire des mobilisations homosexuelles nord-américaines et ouest-européennes des années 1970, qui tend à se diffuser et à s'internationaliser depuis une quinzaine d'années. Dans la continuité des travaux de Jasbir K. Puar (Puar 2012), Marien Gouyon critique toute forme d'essentialisation de l'identité homosexuelle et d'instrumentalisation ([homo]nationaliste, impérialiste, xénophobe ou classiste), en proposant une approche constructiviste et situationnelle des processus d'identification à l'homosexualité et des rapports de domination qui en découlent au sein même du 
groupe étudié et en dehors. En cela, il s'inspire également du courant états-unien du black feminism en s'intéressant aux normes sociales auxquelles se réfèrent les personnes pour s'identifier comme homosexuelles, à l'imbrication de critères de classe, de race et de genre à l'origine de hiérarchies, de rapports de pouvoir, et à la production de souscatégories identitaires plus ou moins légitimes au sein des groupes. À contre-courant d'une vision victimaire, il envisage les identités homosexuelles casablancaises comme autant de pratiques de résistances quotidiennes aux dominations, stigmatisations, délégitimations et pressions à la fois internes et externes aux groupes qu'il a étudiés. L'anthropologue analyse ainsi pas à pas les dimensions sociales, économiques, spatiales, situationnelles, sexuelles, genrées et raciales de ces identités "en mouvement », en s'intéressant « aux contextes dans lesquels (...) [elles] se découvrent, se racontent et les actes qu'elles engagent » (p. 16). À partir d'une enquête ethnographique menée entre 2009 et 2011 auprès de 41 hommes âgés de 16 à 40 ans, il montre alors que les manières de se dire et de s'identifier comme homosexuel à Casablanca sont multiples, concernent toutes les classes sociales et sont consubstantielles aux espaces, aux situations, aux parcours personnels, et aux socialisations individuelles et collectives des individus qu'il a rencontrés.

\section{Résistances spatiales et langagières}

2 Le premier chapitre de l'ouvrage s'intéresse aux usages du langage et des espaces de la ville par les enquêtés, et à leurs rôles dans le façonnement de leurs identités. Dans la continuité des travaux ayant étudié les formes de subversion du langage ou de retournement du stigmate (Goffman 1975; Eribon 1999) l'auteur montre dans un premier temps, que les catégories mobilisées par les personnes pour se définir renvoient à de multiples significations et sont le lieu de détournements et d'adaptations de catégories du langage courant. C'est notamment le cas du terme zamel, équivalent d'une insulte envers les hommes non virils (par exemple non mariés) dans le sens commun, qui est réinterprété et "ironisé " par certains hommes concernés pour s'identifier. Le terme loubia, titre de l'ouvrage, équivalent du mot « haricot » en darija, l'arabe parlé marocain, est également utilisé pour désigner les hommes ayant des attitudes et gestuelles associées à la féminité et qui se considèrent comme homosexuels. L'usage de ces mots prend ainsi le rôle de codes au sein des groupes de personnes concernées et sont des moyens d'exister, de communiquer et de « circuler au sein d'une structure sociale qui les invisibilise et les violente psychologiquement et physiquement » (p. 29). Dans un second temps, l'auteur analyse le rôle de l'occupation d'espaces de la ville de Casablanca par les personnes dans la formation de leurs identités, mais également dans l'intériorisation et la production de normes afférentes. Ces lieux (de drague, de sociabilité ou de consommation sexuelle) ont pour caractéristique, selon l'anthropologue, la mixité sociale des personnes qui les fréquentent. Il s'agit par exemple de cafés, de douches publiques, de parcs ou de plages non officiellement reconnus, peu nombreux, mais fréquentés par un grand nombre des hommes se définissant comme homosexuels, toutes classes sociales confondues. Ces espaces de sociabilité sont présentés comme une des étapes à part entière d'un processus identificatoire ancré dans le quotidien et fluctuant selon les lieux et les situations. Plusieurs "cliques" s'y forment au gré des espaces, des proximités interindividuelles liées à l'âge, la fréquentation de certains lieux au détriment d'autres, les quartiers d'origine, etc. La constitution de ces groupes de personnes s'identifiant 
comme homosexuelles est saisie comme un des moyens de faire circuler et de "tester les identités en construction » (p. 53), de donner « corps au groupe de pairs» (p.54) et parfois même de se visibiliser dans l'espace public selon les situations. Dans un contexte où il est difficile et rare de disposer d'espaces privés où vivre et affirmer son homosexualité, ces lieux - dont les usages sont mouvants, situationnels et non institutionnels - sont analysés d'un point de vue foucaldien comme des « hétérotopies de déviation " où "se construisent, se questionnent, se conservent, se catégorisent " (p. 59) et s'expriment ces identités. La participation de l'auteur à certaines de ces cliques et la fréquentation de ces espaces durant son ethnographie lui permet d'analyser le travail de catégorisation des identités homosexuelles au sein des groupes. En effet, ces espaces de mixité sociale sont propices à la construction de normes, de hiérarchies et de figures repoussoirs au sein des groupes à partir de critères qui croisent notamment la classe sociale, le genre et la sexualité et qui stigmatisent les classes populaires. Il analyse par exemple les catégories de «blédard» ou de «beldi » qui mêlent un certain nombre d'éléments permettant à ceux qui les utilisent de déprécier les classes les plus pauvres, tels que : une homosexualité qui ne serait pas assumée - et donc critiquée comme non identitaire - violente, de personnes non éduquées de classe populaire ou renvoyant au travail du sexe et à l'efféminement. Ces étiquetages «fantasmés » sont des constructions sociales qui, plutôt que de révéler les conditions et pratiques (sociales, sexuelles, économiques...) effectives des personnes, sont surtout des moyens de se distinguer des autres et de se légitimer au sein des groupes. Ce travail de redéfinition sexuelle des classes sociales produit alors une naturalisation des différences de classe à l'encontre des classes populaires que les hommes qui en proviennent apprennent au cours du temps à déjouer et à subvertir.

\section{Espaces virtuels, « lieux intérieurs » et confrontations normatives}

3 Internet joue également un rôle important dans le développement de sociabilités et de subjectivités homosexuelles, ce que l'auteur s'attache à montrer dans le second chapitre. En effet, la fréquentation des réseaux sociaux numériques (forum de discussions, chat, Facebook...) est analysée comme une des premières étapes de la construction de subjectivités homosexuelles. Marien Gouyon montre que pour beaucoup de ses enquêtés, Internet fut un moyen de "mise en mot émotionnelle " (p. 64), de briser un isolement, de se renseigner sur les affects et désirs naissants et d'échanger ses expériences de manière anonyme. Producteur de «communautés momentanées » (p.77), l'espace virtuel permet souvent les premières sociabilités qui conduisent, par la suite, à des rencontres physiques et à la constitution des « cliques ». En définitive, Internet tout comme l'occupation d'espaces intérieurs et extérieurs de la ville de Casablanca sont associés par l'auteur aux "lieux intérieurs » analysés par Jean Noël Ferrié ${ }^{1}$, en tant qu'espaces occupés de manière "temporaire et [qui] donne[nt] l'occasion de se présenter à autrui sous des traits choisis qui ne cadrent pas nécessairement avec l'espace public légitime » (p. 95). L'anthropologue termine son second chapitre par une mise en perspective de ces constructions identitaires locales confrontées aux actions d'une association pour la reconnaissance des droits des homosexuels au Maroc. À travers la description d'une réunion organisée par cette association il montre comment les logiques de visibilité, de libération et de lutte 
politique portées par cette structure entrent en contradiction avec les pratiques quotidiennes de résistances mises en œuvre par les hommes enquêtés. À la suite des travaux portant sur les effets de l'intervention d'organisations de lutte pour les droits dits LGBT sur le continent africain notamment (Lorway 2015), il montre ainsi que par ses actions, l'association participe à produire une vision victimaire et apolitique des personnes concernées qui annihile leurs identités et leur confère le rang de "subalternes ». En plus d'entrer en décalage avec la plupart des manières locales de se dire et de se penser homosexuel, la structure empiète sur certaines formes de résistances développées localement, à travers la reprise d'un forum Internet par exemple. Son action participe par ailleurs à entretenir une image stéréotypée qui associe l'identité homosexuelle au militantisme politique et au monde occidental.

\section{Subjectivations homosexuelles : normes genrées et écarts pratiques}

4 Dans le troisième et dernier chapitre, le chercheur revient en détail sur les parcours d'identification et de subjectivation des homosexuels qu'il a rencontrés. Dans un premier temps, il aborde les différentes contraintes (sociales, familiales, économiques, religieuses) auxquelles sont confrontés ces hommes dans leur "devenir homosexuel » et l'impact de leurs socialisations sur la construction de leurs identités, de l'étape initiale de la prise de conscience de leur « subalternité » à celle de l'affirmation de leur homosexualité. Il met notamment en lumière la place primordiale du «tacite », ou du non-dit, analysé comme "forme d'affirmation" et "soubassement identitaire des homosexualités à Casablanca » (p. 215). Rappelant alors combien, selon les situations, le silence peut disposer d'une «force performative»(Broqua 2009), "ce sont les références biographiques attenantes aux contextes socio-économiques et à ces changements qui signifient ce que parler veut dire, c'est-à-dire à quel moment la parole peut faire partie du possible» (p.129). Ce moyen de gestion et d'adaptation aux contraintes et aux oppressions auxquelles sont confrontés les hommes concernés fait également office de pratique de résistance quotidienne qui leur permet de faire tenir leurs différentes appartenances et obligations sociales. Dans un second temps, l'auteur revient en détail sur les stratifications et hiérarchisations entre les homosexualités. Il montre encore une fois combien toutes se font au détriment des hommes efféminés qui sont associés aux classes populaires et qui sont perçus comme déviants vis-à-vis des normes sociales majoritaires de masculinité, reprises et valorisées dans les discours circulant chez les hommes qui s'identifient comme homosexuels. À cet égard, il s'intéresse notamment à deux éléments de leurs vies sexuelles et affectives que sont les constructions sexe/genre dans les pratiques sexuelles et les rapports entre plaisir sexuel et violence. Il montre ainsi que les différents critères mobilisés pour catégoriser et dévaloriser ceux qui sont considérés comme "efféminés" (rôle sexuel passif et soumis, gestuelle, apparence, scripts sexuels associés au désir, etc.) participent à reproduire la norme hétérosexuelle et la domination masculine présente dans la société marocaine comme ailleurs, et s'impose à tous comme une norme avec laquelle bricoler, s'ajuster ou simplement se positionner. Il construit alors un « continuum de positions » dans lequel se placent à la première extrémité « des individus qui cherchent avant tout à unifier par le plaisir sexuel, deux sexes biologiques masculins dont le genre masculin est visible sur le corps"(p.193-194). L'autre extrémité quant à elle regroupe des 
hommes «dont l'identification homosexuelle ne repose pas sur une stricte correspondance entre sexe et genre. Le sexe ne contraint pas le genre au travers de l'hexis corporel et le plaisir sexuel n'est pas obligatoirement dépendant du genre incorporé du partenaire» (p.194). Dans la pratique, les hommes circulent entre ces modèles et en jouent en fonction de leurs appartenances sociales et de leur " conscientisation des rapports de classe» (p. 194). Les hommes de classes populaires, qui pâtissent le plus de ces constructions et hiérarchisations, sont également ceux qui naviguent le plus entre ces modèles et qui, « de par des conditions socio-économiques particulières, pensent l'importance de la norme de genre dans la sexualité comme secondaire » (p. 214). Ils s'inscrivent ainsi plus souvent dans la seconde extrémité du continuum de positions proposé par l'auteur et développent des pratiques de résistance autant internes au milieu des homosexuels, qu'externes.

5 À l'aide d'un matériau ethnographique dense et diversifié, auquel l'auteur nous donne accès à différents moments de son livre à travers des extraits de notes d'observations et d'entretiens particulièrement riches, Marien Gouyon propose une analyse fine d'un objet encore trop peu étudié dans le champ des recherches en sciences sociales sur les masculinités et les homosexualités. Cet ouvrage est un précieux outil pour toutes celles et tous ceux qui souhaitent comprendre en détails l'imbrication des différents champs du social dans la construction et le développement des identités homosexuelles en contexte urbain marocain. Il en propose une lecture dynamique dont il met en avant le caractère «situationnel » et non figé, où « chaque situation se dote d'une politisation éphémère, individuelle et/ou collective" (p.218), en marge des logiques d'interpellations de l'État classiquement étudiées. En ce sens, Ana Loubia répond amplement à la volonté de l'auteur «de réinscrire la liberté dans la pratique des individus » (p. 15).

\section{BIBLIOGRAPHIE}

BROQUA, Christophe. 2009. "Une découverte scientifique récente : l'homosexualité en Afrique », in L'objet homosexuel : études, constructions, critiques, Cazier, Jean-Philippe (éd.). Mons : Sils Maria, 87-97.

ERIBON, Didier. 1999. Réflexions sur la question gay. Paris : Flammarion.

FERRIE, Jean-Noël. 1995. « Lieux intérieurs et culture publique au Maroc » Politix (31) : 187-202.

GOFFMAN, Erving. 1975. Stigmate. Paris : Editions de Minuit.

LORWAY, Robert. 2015. Namibia's Rainbow Project. Gay rights in an African nation. Bloomington : Indiana University Press.

PUAR, Jasbir K. 2012. Homonationalisme : la politique queer après le 11 septembre 2001. Paris : Éditions Amsterdam. 
NOTES

1. Jean Noël Ferrié est un politiste de l'action publique, directeur de recherche au CNRS, spécialiste du Maroc. Il développe la notion de " lieux intérieurs ", notamment dans " Lieux intérieurs et culture publique au Maroc, Abstract », Politix, 1995, nº 31, p. 187-202 (1995).

INDEX

Thèmes : Actualités

Mots-clés : homosexualités masculines, Maroc, constructions identitaires

Keywords : male homosexuality, Morocco, identity building

\section{AUTEURS}

\section{LUCILLE GALLARDO}

Doctorante en sociologie, Université Paris Nanterre, laboratoire SOPHIAPOL. 